\title{
Medicina Tradicional Ancestral: Perspectiva de sanación de Pauka/krisi Siknis
}

\author{
Traditional Ancestral Medicine: Pauka / Krisi Siknis Healing Perspective
}

\author{
Serafina Espinoza Blanco ${ }^{1}$ \\ María Sol Pastorino ${ }^{2}$
}

\section{Resumen}

El Pauka/krisi siknis, consiste en un problema de salud, de aparición repentina, afecta principalmente a jóvenes, a pesar de remontarse a épocas antiguas, aún se desconocen las causas, lo que impide que el personal de salud pueda manejarlo como el resto de patologías.

Con el propósito de analizar la experiencia del proceso de sanación del pauka/ krisi siknis, desde la medicina tradicional ancestral, se describen los conocimientos, saberes y sentires de la población indígena miskitu sobre este problema de salud. La estrategia metodológica consistió en la revisión documental, que involucró la revisión de estudios científicos y publicaciones. En su mayoría realizados por antropólogos extranjeros donde resaltan los investigadores de las universidades de Estados Unidos de América.

Este problema de salud, debe de ser valorado y atendido desde una perspectiva de salud intercultural para que de esta manera responda a la armonización entre la medicina convencional/biomedicina y la medicina ancestral. Un abordaje de esta naturaleza podría conllevar a que se eviten brotes masivos, controlando de manera oportuna la cantidad de personas afectadas.

Palabras clave: Pauka/krisi siknis; medicina tradicional ancestral; sanadores; brotes; indígenas miskitus.

\section{Abstract}

The Pauka / Krisi Siknis consists of a health problem, of sudden appearance, it affects mainly young people, despite going back to ancient times, the causes are still unknown, which prevents health personnel from being able to handle it like the rest of pathologies.

\footnotetext{
1 MSc. En enfermería con mención en Docencia, Investigadora, docente de la URACCAN Correo: serafinaespinoza@gmail.com ORCID: https://orcid.org/oooo-0002-0124-5531

2 Doctora en Ciencias Sociales. Centro de Investigaciones y Estudios de la Salud (CIES-UNAN-Managua). lic.msolpastorino@gmail. com ORCID: https://orcid.org/oooo-0002-5368-8457
}

Recibido: 08/01/2021 - Aprobado: 05/02/2021 
In order to analyze the experience of the healing process of the pauka / krisi siknis, from the traditional ancestral medicine, the knowledge, knowings and feelings of the indigenous Miskitu population about this health problem are described. The methodological strategy consisted of the documentary review, which involved the review of scientific studies and publications. Mostly carried out by foreign anthropologists where researchers from universities in the United States of America stand out.

This health problem must be valued and addressed from an intercultural health perspective so that in this way it responds to the harmonization between conventional medicine / biomedicine and ancestral medicine. An approach of this nature could lead to massive outbreaks being avoided, by controlling the number of people affected in a timely manner.

Keywords: Pauka / Krisi Siknis; Tradicional Ancestral Medicine; Healers; Outbreak; Miskitu Indigenous

\section{Introducción}

El pauka/krisi siknis, consiste en el desequilibrio de la salud principalmente de los jóvenes. Entre la población indígena miskitu es un problema común con el que se ha lidiado desde épocas ancestrales. De acuerdo a su cosmovisión, este desequilibrio es originado por fenómenos sobrenaturales. El medio para la sanación desde siempre ha sido a través de la medicina ancestral con la intervención de sanadores tradicionales.

La medicina ancestral es en ocasiones el único medio para la sanación de diversos problemas de salud en esta población, es una práctica milenaria que se viene transmitiendo de generación en generación. Esta medicina incluye la utilización de plantas medicinales, aceites de origen natural y animal, así también de algunos minerales.

Este artículo realiza un recorrido histórico del pauka/krisi siknis para que constituya la comprensión a nivel general de sus características desde la cosmovisión ancestral. Grisi siknis o también Krisi siknis (en lengua misquita "locura de la selva", aunque las palabras derivan probablemente del inglés), también conocido como grisi munais, chipil siknis y nil siknis, es un síndrome contagioso y legado culturalmente a la etnia del pueblo Misquito en América Central.

Esta afectación de la salud se caracteriza por ocasionar brotes de considerable magnitud, como principales signos y síntomas los afectados presentan: pérdida de la consciencia, trance o convulsiones, visiones espirituales malignas, sueños de conexión espiritual, enojo, dolor de cabeza, mareos, conducta alterada.

Con el propósito de Interpretar y comprender la experiencia del proceso de sanación del pauka/krisi siknis desde la medicina ancestral, como parte de los conocimientos 
que tienen los indígenas miskitus, se ha realizado la revisión de la literatura existente al respecto.

Cabe mencionar que, siendo un problema de salud, aún no comprendido a profundidad por el personal del Ministerio de Salud, la responsabilidad siempre recayó en las familias de las personas afectadas, por medio de la intervención de los sanadores tradicionales quienes utilizaron la medicina ancestral para el restablecimiento de la salud de los afectados.

\section{Revisión de Literatura}

\section{Memoria histórica del pauka/krisi siknis}

El pauka/krisi siknis, es un problema que desde tiempos remotos ha venido afectando la salud en los pueblos indígenas de la Costa Caribe Nicaragüense. Por ende, resulta oportuno describir que este problema de salud siempre fue y ha sido resuelto desde el ámbito comunitario y familiar por parte de los sanadores tradicionales/sika lan uplika nani, quienes tienen dominio de la medicina tradicional ancestral.

Esta medicina desde tiempos antiguos ha sido tildada como brujería/ hechicería por parte de escépticos, como resultado los sanadores tradicionales intervienen de manera clandestina. Este importante conocimiento se transmitía y se transmite aún de manera oral.

Debido a los severos efectos que ocasionaba el pauka/krisi siknis en las familias y comunidades, llamo la atención de los primeros colonizadores que llegaron a estas tierras vírgenes, donde los indígenas miskitus sobrevivían en medio de la naturaleza. Cabe agregar entonces, que fueron ellos los primeros en documentar lo que observaron sobre pauka/krisi siknis. Sobre la base de las consideraciones anteriores, describo algunas evidencias documentadas:

Dennis P., Antropólogo de la Universidad de Texas, EEUU, estudió sobre el fenómeno del Grisi Siknis durante1978, 1979, 1999 y 2000, él, cita a Charles Napier Bell, un inglés que vivió en la Costa Atlántica de Nicaragua y realizó una publicación en 1862, en la revista británica: Journal of the Royal Geografical Society, el artículo titulado: Remarks on the Mosquito Terrytory, its climate, people, Production, en la que describe ataques de grisi siknis y su curación. Esta publicación indica que el pauka/krisi siknis es un problema de salud que ha estado presente en esos pueblos desde épocas ancestrales.

Cabe agregar que, desde tiempos remotos, a la actualidad, el origen de esta enfermedad ha estado relacionado a fenómenos sobrenaturales donde intervienen espíritus 
malignos y personas mal intencionadas, según han expresado siempre los sanadores tradicionales/sika lan umplika nani, entre ellos: sukias, curanderos, prapit, spirits.

En este sentido es importante considerar lo que expresa Pérez C. (2002). En la cosmología miskitu, las enfermedades físicas y mentales, los accidentes y la muerte son todas condiciones humanas resultantes de la posesión espiritual o la magia. La esfera de la naturaleza es también el espacio poblado por los espíritus. Estos manifiestan rasgos humanos en su conducta, y muestran emociones como amor u odio. Todo contacto entre humanos y espíritus que ocurra sin el control adecuado, resulta en enfermedad (p.122-123-127-132).

Los misquitos dependían del sukia, el chamán de la comunidad para controlar a los lasas/espíritus El sukia, era considerado un exorcista, un adivino y un curandero (Helms; 1971,182-186). Si bien la iglesia Morava ha luchado contra esta creencia, los sukias existen todavía. Las doctrinas de la iglesia Morava y otras religiones han suplantado en gran medida las creencias misquitas tradicionales.

Pérez C. (2002) refiere que la restauración de la salud física y desórdenes emocionales o mentales requiere de la expulsión del espíritu y la adopción de medidas que prevengan su reingreso al cuerpo del paciente (p.132).

La vida de la población miskitu ha transcurrido en torno a su cosmovisión y la naturaleza, es evidente que, en este marco, el tema de los espíritus malignos sea parte de la vida y de la cotidianidad. Son reconocidos diversos espíritus que se encuentran en las profundidades de ríos, montañas, llanos, cerros, y en el cosmos. Estos espíritus si son invocados por las personas pueden hacer presencia causando problemas en la salud como por ejemplo pauka/krisi siknis que causa principalmente pérdida de conciencia y trance/ataques.

Los hallazgos de Pérez, C. (2002) describen que, las personas afectadas por grisi siknis o blakira, presentan las siguientes señales a nivel físico y mental.

Los ataques seguían un patrón en el que primero una muchacha perdía el control de sí, y luego le seguían otras que también perdían el control de sí. Seguidamente las jóvenes se tornaban serias, dejaban de hablar, empezaban a perder el control de sus cuerpos, gritaban, rodaban por el suelo y repentinamente se ponían de pie, agarraban un leño o un machete, y comenzaban a correr. Corrían hacia el río, los matorrales y el cementerio; daban muestras de una fuerza descomunal, y podían causar daño a quien intentara detenerlas o las imitara. Después de un tiempo, las vencía el cansancio o eran amarradas por sus parientes. Entonces, yacían con los ojos abiertos y la mirada desenfocada, exhaustas y sin poder dormir (Ibíd., p.23o). 
También demostró que, los miskitus consideran un hechizo o maleficio, es necesario para que blakira se dé en una comunidad. (Pérez; 2002, p.254-157).

Venegas (2016), Davis et.al. (2006), Fagoth et.al. (1988), Jamieson (2013), Von Houwald (2003), Wedel (2012), coinciden en sus estudios sobre pauka/krisi siknis, donde observaron y recopilaron información por medio de entrevistas a personas afectas y actores claves, las mismas alteraciones que presentan las personas afectadas a nivel físico y mental por causa de esta enfermedad. Incluyendo la sanación por la vía de la medicina tradicional ancestral.

De acuerdo a registros históricos, la cultura miskitu siempre fue influenciada por extranjeros; sin embargo, parece ser que la de mayor influencia fue la cultura africana, con quienes mantuvieron acercamientos que incluyó matrimonios entre mujeres indígenas y hombres africanos. Como resultado de la influencia africana, se combinó la práctica de la medicina tradicional indígena miskitu con la africana.

La medicina tradicional ancestral ha sido una de las principales alternativas de sanación para diversos problemas de salud en los pueblos indígenas miskitus, incluyendo el Pauka/krisi siknis. La medicina convencional no ha logrado curar esta enfermedad catalogada como de origen sociocultural.

Un elemento importante es que La organización social miskita aún conserva la esencia de su cultura a pesar del contacto con el mundo exterior, pero se presenta el riesgo de ir desapareciendo así lo expresan los ancianos "todas nuestras costumbres buenas se están exterminando los jóvenes se van afuera y vienen con ideas distintas". (Espinoza, 2001, p. 30).

\section{Materiales y métodos}

Se revisó la literatura existente sobre pauka/krisi siknis realizando búsqueda en las siguientes bases de datos: repositorio de la URACCAN, EBSCO, Scielo, PubMed y otros sitos virtuales, como también publicaciones en físico. Además de la revisión de la literatura, se recopilaron testimonios de personas afectadas y de sanadores tradicionales. Este artículo es de carácter descriptivo, analítico y reflexivo. La revisión de las investigaciones evidencia los hallazgos relevantes a partir de lo que narra la población afectada, de familiares y de sanadores tradicionales/sika lan uplika nani.

\section{Resultados}

En este acápite se describen los hallazgos, agrupados en diferentes categorías de análisis temáticas. 


\section{Creencias y tradiciones entre los miskitus}

García C. (2002) describe que, para fines del siglo XVII, los africanos fueron asimilados a la sociedad de acogida, especialmente en la zona circundante a Cabo Gracias a Dios, (esta comunidad está ubicada en el municipio de Waspam río Coco), en la que los valores y prácticas provenientes de África se van a fundir con los indígenas. La adopción por los miskitu de individuos de diferentes orígenes (y con diferentes lenguas) es uno de los factores decisivos que promociona los intercambios interculturales (p.449-450).

En este sentido, como investigadora indígena miskita y en base a mi experiencia de varios años en procesos de acompañamiento a sanadores tradicionales de la Costa Caribe Norte de Nicaragua, considero que en estos tiempos "modernos" la medicina tradicional y los diversos rituales para los procesos de sanación que combinan la utilización de plantas medicinales y la espiritualidad indígena, continúan siendo una opción más para mantener una buena salud.

Los sukias y otros tipos de sanadores tradicionales en épocas pasadas, también tenían habilidades de la clarividencia y también tenían la habilidad de comunicarse con el mundo espiritual. En estos tiempos contemporáneos, son muy pocos los sukias que han quedado en las comunidades más recónditas y alejadas de la región.

El mundo espiritual es efectivamente una parte fundamental de las creencias de este pueblo con varias experiencias vividas. No se ven a simple vista, pero, se pueden conectar con los humanos principalmente a través de los sueños, en otras ocasiones se siente la presencia de los entes espirituales, y en raras ocasiones pueden materializarse ante algunas pocas personas. Consiste en la conexión con otras realidades que se encuentran en el entorno de la naturaleza, en el cosmos. Lo sobrenatural ha sido muy poco estudiado.

Diversos acontecimientos sobrenaturales acontecidos a través de los tiempos, han hecho de que la población indígena miskitu, perciba la salud y la enfermedad desde otro punto de vista comparado a otras culturas, principalmente la occidental. Este hecho no significa que sean atrasados, sino que, al contrario, estos conocimientos deben de ser evidenciados y potenciados por medio de las investigaciones científicas.

\section{Nociones y comportamientos asociados al pauka/krisi siknis}

El pauka/krisi siknis, se caracteriza por causar desequilibrio en la salud de las personas, principalmente entre jóvenes, por lo general afecta la parte emocional o mental, sin que la persona tenga historial médico de padecer una enfermedad de esta causa. Son personas sanas y saludables al momento de presentar los desequilibrios en su salud. 
En ocasiones también podrían producirse afectaciones a nivel físico, asociado más que todo a lesiones físicas al momento de entrar en trance. Este es un problema de salud de considerable magnitud, debido a los brotes que aumentan rápidamente, lo que causa un alto grado de estrés, desesperación y angustia a nivel familiar.

Los estados de trance o ataques son constantes, pueden durar varios minutos, ya sea entre 20 ó 40 minutos. La persona afectada no puede quedar sola, ya que está mentalmente descontrolada, puede pasar mucho tiempo con ataques repetitivos, se ha observado en esta etapa una fuerza descomunal, y lucha para querer salir corriendo. Si lo logra, se torna muy difícil tener el control pues tiene una gran velocidad, la persona puede correr con los ojos cerrados y no tropezar y caer.

La persona afectada se vuelve muy agresiva, está enojada sin causa alguna, se observa transformación en la fisonomía física de la cara, tiene los ojos diferentes con una mirada penetrante, grita, parece comunicarse con alguien que sólo el afectado puede ver. Para lidiar con la situación, las familias deben de estar bien preparadas, y al pendiente, lo que significa un cuidado permanente. En ocasiones tienen que inmovilizarlos, amarrando los miembros inferiores y superiores para controlarlos. No le llega ningún medicamento, aunque sean sedantes.

Estas personas desde el punto de vista de la biomedicina están sanas, pues en algunos brotes más recientes se les han realizado chequeos médicos en la búsqueda de alguna enfermedad, enviando incluso pruebas de laboratorio, no encontrándose con ninguna otra patología o sea son jóvenes sanos y saludables.

Algunas familias recurren donde los líderes religiosos para que hagan oraciones por los enfermos y ponen en manos de Dios a las personas afectadas. Los resultados de las oraciones podrían clasificarse, desde leves a moderadas, y en algunas ocasiones como efectivas, ya que calma y controla el estado de trance, la gente afectada puede descansar un poco más, incluso pueden dormir y alimentarse. Pero siempre se tiene que estar pendiente, vigilando, ya que los estados de trance pueden volver a aparecer.

Los efectos de las oraciones en algunos casos no duran por mucho tiempo. Sin embargo, a las familias les da tiempo de buscar a un sanador tradicional. Esta situación afecta a todas las familias e incluso, si la cantidad de afectados son muchos se involucran a los líderes comunales para que de manera conjunta se organicen para hacerle frente a la situación.

Por lo general se busca apoyo de hombres y mujeres, sea parte de la familia o no, para apoyar con los cuidados. En brotes epidémicos, la comunidad se paraliza, se selecciona un lugar, sea una iglesia, casa comunal o escuela para concentrar ahí a todos los afectados. 
Tomando en cuenta la difícil experiencia que ha vivido la población miskitu desde épocas milenarias, es común que relacionen algunas enfermedades de origen biológico, con desequilibrios originados por fuerzas sobrenaturales o enfermedades de origen sociocultural. En este sentido se debe de poseer la habilidad tanto por parte de las familias, como de los líderes comunales y territoriales que conlleven a la interpretación del verdadero origen del pauka/krisi siknis, por lo tanto, los sanadores tradicionales deben de tener experiencia y capacidad. También se sugiere involucrar al personal del Ministerio de Salud.

\section{¿Quiénes son los sanadores tradicionales?}

La población indígena miskitu, mantiene su propia idiosincrasia. En este marco la salud es fundamental, consideran que existen diversas maneras de enfermarse. Las de origen biológico causado por microorganismos patógenos y que la biomedicina los atiende, también están los desequilibrios de la salud ocasionados por espíritus malignos presentes en el entorno de la naturaleza, ya sea en las montañas, los cerros, los llanos, en los ríos, lagunas, en el mar, en el cosmos.

Con el propósito de mantener la buena salud entre la población es común encontrar sanadores tradicionales, algunos fueron elegidos por personas mayores en el seno de la familia considerando los dones que posee la persona, otros fueron desarrollando sus conocimientos a través del acompañamiento de otro sanador de mayor experiencia, y en épocas antiguas, esta sabiduría milenaria se daba por medio de la intervención de los espíritus, ellos eran los sukias.

Conzemiuz (1932), citado por Von Houwald (2003), describe las múltiples funciones de un sukia: curandero, doctor, vidente, conjurador, mago, encantador, adivino, exorcista, maestro, guía, consejero, depositario de las tradiciones de las tribus. También se le agrega a los spirit y el obeah, estas dos categorías provienen de tradiciones africanas. (p.,139. y 390-391). Probablemente cada poblado sumu o miskitu tenía su propio chamán. Sin embargo, siempre se habla de "sukias", cuyo radio de influencia obviamente se extendía más allá de su propia localidad. Los sukias tenían en todo caso la fama de tener importancia aún más allá de sus respectivos poblados. (Ibíd).

Fagot et al. (1998), describe a los sanadores tradicionales de la siguiente manera:

Son los principales protagonistas para el proceso de sanación, comprende diferentes actores como la partera, el sobador, el yerbero, el curandero y sukia. Son muy celosos al guardar para ellos o un pequeño círculo restringido los secretos de las plantas con que curan. Una costumbre que ha logrado sobrevivir a los embates del tiempo es el ritual de agradecimiento a la planta que va a utilizar, dejando una moneda en la raíz. De acuerdo a las categorías poseen secretos más profundos (p.21). 
Por lo general, estos conocedores de los misterios y misticismo adquieren sus conocimientos a temprana edad, algunos son seleccionados por sus padres o familiares adultos mayores más cercanos, los que se encargan de entrenarlos a través del sistema oral y la observación, llegando a memorizar los distintos rituales.

En este sentido, los conocimientos tradicionales sobre la salud en muchas ocasiones resultan oportunos, por ende, no debería de ser desvalorizada sino, más bien aprovechada para el restablecimiento de la salud. Esto debido a la inequidad que existen en los sistemas de salud.

En ocasiones por la situación geográfica, económica y cultural, sumado a ello los niveles de pobreza, a muchas familias principalmente de zonas muy alejadas se les dificulta acudir a las unidades de salud pública, mucho menos un sistema de salud privado. Estas realidades los ha llevado a buscar en la naturaleza, en la medicina tradicional ancestral recursos para la sanación.

En este sentido, Romero et al., (2007) refiere: "en cualquier comunidad miskita, existe la tradición de curar por medio de plantas medicinales. Este conocimiento se trasmite de una generación a otra como parte de la cultura. Las enfermedades que se adquieren en las montañas, llanuras, ríos y lagunas, causados por malos espíritus, no son para curarse en las clínicas". (p. 279).

Los hallazgos de Romero, conlleva a la comprensión de los conocimientos tradicionales y efectivamente sobre la sanación de los diversos problemas de salud que tiene este pueblo indígena, sean estos de origen natural, como sobrenatural.

De acuerdo con Fagetty (2011), la medicina tradicional es parte de ese saber que le ha permitido a la humanidad sobrevivir, enfrentar lo que desde siempre ha amenazado la integridad física, emocional y espiritual: el infortunio, la enfermedad y la muerte. Todos los pueblos que han habitado la tierra poseen una peculiar visión de estos sucesos, la cual se expresa en un discurso que explica su origen, devela sus causas y, sobre todo, revela cómo el individuo puede hacerles frente. La desgracia, la enfermedad y la muerte son interpretadas a partir de una representación del mundo y de todo lo que contiene, que explica su existencia y la vida misma, ordenando todo acto y prácticas humanas (p.137).

Sin embargo, aun en estas épocas contemporáneos, lo que plantea Fagetty, sobre estos valiosos conocimientos tradicionales, no han hecho eco en la comunidad científica, quienes más bien han venido a contribuir en la desaparición acelerada de estas efectivas practicas milenarias, obviándolas cada vez más, incluso por la misma población indígena debido a la aculturación. Esta realidad se plantea a continuación. 


\section{Misterios y sanación: historicidad de sobrevivencia}

Los siguientes elementos son considerados claves para que surtan efecto los rituales de sanación:

La hora en que se va a cortar la planta medicinal o la parte que se va a utilizar sean estas: flores, hojas, raíces, cortezas, látex o frutos. La ubicación de las mismas en base a los cuatro puntos cardinales, junto con las oraciones.

Los tipos de aplicaciones, ya sea a través de baños, sobados, cataplasma, sahumerio/pukni, limpias tradicionales, ya sea a nivel de los hogares o en toda la comunidad. Se utilizan plantas medicinales, cortezas, nidos de algunas especies de animales, ya utilizados y abandonados, que se encuentran en los árboles, resina o látex del árbol de pino u otro árbol misterioso o de su corteza.

\section{Pauka/krisi siknis una perspectiva desde la cosmovisión miskitu}

De acuerdo a Martínez (SF), en el lenguaje biomédico el Grisi Siknis se sitúa dentro de las narrativas de salud mental, parte de los trastornos mentales disociativos culturales, junto con otros "síndromes ligados a la cultura", característico de algunos pueblos indígenas, como el pibloktoq o la histeria ártica de los inuit que viven dentro del Círculo Polar Ártico, la brujería "frenesí" de los navajo, el chakore de los ngawbere del norte de Panamá y algunas formas de amok de Indonesia y Malaysia, entre otros (GLADP 2003, DSM IV 2000 y WHO 1993).

Según estas fuentes, debido a sus características de un estado como de trance, altos niveles de actividad, agotamiento y amnesia posterior de los episodios, también es comúnmente conocido como síndrome del "corredor" o del "mareo". (ibid,p. 5).

El relato de Dennis (1981) se refiere a cuestiones relacionadas con el estrés, en experiencias de la vida, por otro lado, Wedel (2012) destaca la necesidad de comprender el contexto social, personal, ambiental y el papel de la cultura en relación con la enfermedad y el sufrimiento. Jamieson (2013), señala las presiones sobre las mujeres jóvenes durante su transición a la adultez.

Wedel, llamó la atención sobre la mirada del grisi siknis desde una perspectiva transcultural, más allá de la clasificación "vinculado a la cultura miskita", hacia una comprensión de éste como una versión miskita de una posesión masiva involuntaria de espíritus.

Los sanadores tradicionales indígenas miskitus, desde siempre han hecho sus planteamientos en el marco de los hallazgos de Wedel, manifestando que pauka/ 
krisi siknis se origina por medio de una compleja situación que involucra espíritus malignos y hechicería.

He aquí lo que desde siempre han dicho los sanadores tradicionales; "este es un problema de salud que se origina de dos fuentes a saber: i) Malos espíritus, que de manera directa invaden el cuerpo de las personas, ii) Hechicería provocada por personas malintencionadas a través de invocaciones espirituales demoniacas.

Esta aseveración, es bien acogida por la población miskitu, ya que además de ser parte de su sistema de creencias, está comprobado que la única fuente de sanación al mal que afecta principalmente a la población joven, es a través de la medicina tradicional ancestral de manejo propio desde esto pueblo.

Sin embargo, la ciencia occidental, aún no comprende los misterios que existen en los conocimientos que tienen los indígenas miskitus sobre la salud y la enfermedad. Esto ha limitado el avance en el campo de la investigación científica, medio por el cual se podrían ir desclasificando los misterios.

Por otro lado, es importante recalcar que esta enfermedad podría confundirse con trastornos mentales de origen biológico, por adicción a las drogas y algunos efectos farmacológicos entre otras causas. Ante esta realidad es importante clarificar que el pauka/krisi siknis está relacionado a un problema de origen sociocultural.

\section{La medicina tradicional ancestral como medio de sanación}

La medicina tradicional tiene una larga historia. Es la suma total de los conocimientos, capacidades y prácticas basados en las teorías, creencias y experiencias propias de diferentes culturas, bien sean explicables o no, utilizadas para mantener la salud y prevenir, diagnosticar, mejorar o tratar enfermedades físicas y mentales. (OMS 2014, p.,15).

Esta forma de atención está próxima a los hogares, es accesible y asequible. Además, es culturalmente aceptada y en ella confían muchísimas personas. La asequibilidad de la mayor parte de las medicinas tradicionales las hace más atractivas en el contexto del vertiginoso encarecimiento de la atención de salud y de la austeridad casi universal (ibíd.).

La medicina tradicional ancestral, la principal fuente de sanación para diversos problemas de salud en los pueblos indígenas miskitus, es fundamental que estos conocimientos se continúen transmitiendo de una generación a través de la oralidad, única vía que aún mantienen las personas de la tercera edad en quienes aún permanece esta sabiduría milenaria. 
A pesar de la historia de asimilación, de inclusión forzada a través de la castellanización y occidentalización; los pueblos indígenas mantienen sus conocimientos, sus propias formas de percibir el mundo y de vivir en él. Es desde esta visión indígena que se establecen diálogos o se confrontan con los conocimientos occidentales. (Giovanna, Pesantes y Rodríguez 2017, p.155).

Abordar el tema de la medicina tradicional ancestral, es complejo ya que se trata de un tema amplio, esta medicina se caracteriza por la aplicación de distintos rituales para distintos procesos de sanación, consiste en la utilización de diversas especies de plantas medicinales, sin embargo, no es imposible la articulación de acciones entre el sistema de salud pública o ministerio de salud.

Las narrativas, tanto orales como escrita de los pueblos indígenas y afrodescendientes de la Costa Caribe de Nicaragua, expresan visiones de mundo y del bienestar que tienen al centro aspectos como la vida comunitaria, la armonía y la espiritualidad, en las narrativas institucionales predominantes sobre la salud de estos pueblos siguen prevaleciendo visiones que fragmentan, instrumentalizan y minimizan los sistemas de salud "tradicional ancestral" (Henríquez, Chacón, Espinoza, 2016),

La población indígena miskitu tiene sus propios conocimientos, costumbres y tradiciones en distintos ámbitos, así también sus particularidades con relación a la salud y la enfermedad. Estas particularidades no provienen de las diferencias biológicas, pero sí de las diferencias socioculturales.

Langdon EJ, Wiik FB (2010, p.178) afirman que, una persona "tiene cultura" cuando tiene formación escolar avanzada, se origina de una familia de alto nivel socioeconómico o conoce las artes y la filosofía. Es usual afirmar que un "buen paciente" es aquel que "posee cultura", cultura suficiente para comprender y seguir las orientaciones y cuidados transmitidos por el médico o enfermero. Este tipo de paciente es contrastado con el "sin cultura", considerado un paciente más "difícil", que actúa equivocadamente por "ignorancia" o guiado por "supersticiones".

Tomado en consideración la afirmación de Langdon y Wiik, se consideró que es una perspectiva positiva para analizar el comportamiento del pauka/krisi siknis partiendo del concepto de que todos tienen cultura, y de que es la cultura que determina estas particularidades. Igualmente sustentan que las cuestiones inherentes a la salud y a la enfermedad deben ser pensadas a partir de los contextos socioculturales específicos en los cuales los mismos ocurren. (ibíd).

\section{Un concepto instrumental de cultura}

La cultura puede ser definida como un conjunto de elementos que median y califican cualquier actividad física o mental que no sea determinada por la biología y que sea 
compartida por diferentes miembros de un grupo social. Se trata de elementos sobre los cuales los actores sociales, construyen significados para las acciones e interacciones sociales concretas y temporales, así como sustentan las formas sociales vigentes, las instituciones y sus modelos operativos. La cultura incluye valores, símbolos, normas y prácticas. La cultura es aprendida, compartida, y estandarizada. (Langdon EJ, Wiik FB, 2010, p.179).

Al encontrarnos con las costumbres presentes en otras culturas, debemos tratar de entender el por qué. De esta forma, evitamos una comprensión etnocéntrica a su respecto, o sea: juzgar a la cultura según nuestros propios valores y clasificaciones del mundo, y no según los que son propios de su cultura (ibíd.).

Siguiendo a Langdon y Wiik, se contrapone el problema de salud milenario denominado por la población pauka/krisi siknis, esta tiene su raíz basada en la cultura de los antepasados miskitus, quienes vivían en una sociedad con principios de comunidad y unidad. De acuerdo a la cultura, era común encontrar sanadores tradicionales, con amplia experiencia en su propia medicina, misma que tenía y aún tiene su base en las plantas medicinales, la espiritualidad y la utilización de algunos minerales. Todos elementos de la naturaleza, que actúan, ya sea de manera separada, como combinada.

Los sanadores tradicionales adquirían sus conocimientos de manera oral, trasmitiéndose de una generación a otra y a través técnicas como: la observación, tocando, fijándose en las características de las diferentes especies de plantas medicinales, sus flores, sus frutos, sus hojas, sus olores, sus formas, tamaños, sus tipos de raíces.

Fundamentalmente, la cultura organiza el mundo de cada grupo social, según su lógica. Se trata de una experiencia integradora y total, de pertenecer y, consecuentemente, formadora y mantenedora de grupos sociales que comparten, comunican y replican sus formas, instituciones, principios y valores culturales. (Langdon EJ, Wiik FB, 2010, p.180).

La perspectiva antropológica requiere que, al encontrarnos con culturas diferentes, no hagamos juzgamientos de valor con base en nuestro propio sistema cultural y pasemos a percibir a las otras culturas según sus propios valores y conocimientos a través de los cuales expresan una visión de mundo propia, que orienta sus prácticas, conocimientos y actitudes. A este procedimiento denominamos relativismo cultural (Ibíd. 181,182).

\section{Relativismo cultural}

Es el que nos permite comprender el porqué de las actividades y los sentidos atribuidos a ellas de forma lógica, sin jerarquizarlos o juzgarlos, pero solamente, y sobre todo, jreconociéndolos como diferentes! (ibíd). 
En este sentido es comprensible que el pauka/krisi siknis, forme parte de la cultura en los pueblos indígenas miskitus, de los males que aquejan a estos pueblos y de la manera de sanación, incluyendo sus rituales y su medicina tradicional ancestral.

\section{Pauka, una visión biomédica desde la psiquiatría y la salud mental}

Hales et al., en el tratado de psiquiatría (1985), en la tabla 41- 4, registra al grisi siknis, como: un síndrome específico de la cultura. Distribución étnica: tribu miskitu de Nicaragua. Cuadro clínico: letargia prodrómica, estado ansioso y depresivo, seguida de agitación, acción de correr aparentemente sin objetivo, finaliza con agotamiento, sueño y amnesia del episodio. (p.1,458).

En el DSM-IV manual diagnóstico y estadístico de los trastornos mentales (1995), describen problema de salud denominado trastorno disociativo de trance y sus características diagnósticas como estados voluntarios y no patológicos son frecuentes y constituyen la gran mayoría de los estados de trance y posesión que se encuentran en diversas culturas. Sin embargo, algunos individuos que entran en estados de trance o posesión, acordes con su marco cultural presentan también síntomas que provocan malestar clínicamente significativo y que podrían corresponder al trastorno que aquí se describe (p.743,744).

En el estado de posesión aparecen una (o más) identidades distintas y alternativas que se presentan con movimientos, recuerdos y actitudes característicos, y con actividades que en este caso sí muestran una cierta complejidad (p. ej., conversaciones coherentes, gestos característicos, expresiones faciales y verbalizaciones específicas de agentes poseedores concretos, acordes con los cánones culturales del lugar). (Ibíd).

Existen considerables variaciones locales en cuanto a edad y modo de inicio. Característicamente, el curso es en forma de episodios agudos de una duración que puede oscilar entre minutos y horas. Se ha dicho que durante el estado de trance el individuo puede mostrar un aumento del umbral sensitivo del dolor, ingerir materiales no digeribles (p. ej., cristal) y experimentar un aumento de fuerza muscular. Los agentes que realizan presumiblemente la posesión son usualmente espíritus naturales (p. ej., espíritus de la muerte, entidades sobrenaturales, dioses, demonios) que a menudo presentan exigencias o demuestran animosidad. Es muy típico que los individuos que sufren estos estados patológicos de posesión encarnen a un número limitado de agentes (uno a cinco) de una forma secuencial y no simultánea. Entre las complicaciones de este trastorno cabe citar intentos de suicidio, automutilaciones y accidentes. (Ibíd, p.,748).

A pesar de que se describe el trance, la posesión y los espíritus, no se evidencia los procesos de sanación que utilizan algunos pueblos, en este estudio solo se consideró a 
la población indígena miskita. Pueblos que desde épocas milenarias han tenido como base sus conocimientos sobre la medicina tradicional, medio por el cual se obtiene la sanación del pauka/krisi siknis.

\section{Conclusiones}

El pauka/krisi siknis, es el causante del desequilibrio en la salud en la población indígena miskitu, principalmente entre jóvenes de ambos sexos, siendo las mujeres más afectadas. Es un problema de salud que ha existido desde tiempos milenarios. Desde esas épocas ancestrales, hasta nuestros tiempos contemporáneos, la sanación ha sido posible con la intervención de sanadores tradicionales, quienes utilizan la medicina tradicional ancestral. Este problema de salud amerita una intervención paralela entre el ministerio de salud y los sanadores tradicionales.

Martínez (SF) indica que, la etnohistoria del Grisi Siknis, está registrado en textos históricos de viajeros que llegaron a estas tierras en época de la colonización, en relatos y narrativas de antropólogos hace ya más de un siglo. Ahora es casi incuestionable que el Grisi Siknis, tiene una larga historia entre las poblaciones miskitas en Honduras y Nicaragua. ; Dennis (1999) y Jamieson (2001) han identificado características de "la histeria miskita" estrechamente relacionadas con Grisi Siknis, en estudios antiguos realizados por Bell (1862) y Conzemius (1932 p.,8).

Registros posteriores como la referencia de Dennis (1999: p.10), sobre la epidemia en Awastara, una comunidad del litoral norte en la Costa Caribe de Nicaragua, hubo más de 60 personas afectadas y Jamieson (2001), quien describe episodios esporádicos en los años noventa en Kakabila y brotes en Raitipura Honduras en 1992 y 1993.

Es importante clarificar el término "histeria miskita", descrita por los antropólogos, quienes hacen referencia a la "histeria colectiva", una enfermedad clasificada y reconocida como un trastorno mental en el CIE 10 (Clasificación Internacional de las Enfermedades y Trastornos relacionados con la Salud Mental realizada por la OMS 10. ${ }^{\text {a }}$ edición). Esta enfermedad es tratada por la medicina convencional por especialistas del área de la salud mental, como psicólogos y psiquiatras.

En el caso de esta enfermedad, se trata de un desequilibrio de la salud que, a pesar de afectar la mente de la persona, no puede confundirse con histeria colectiva, ya que los afectados no presentan signos y síntomas similares.

Es pertinente reconocer, que la ciencia médica o sea los especialistas médicos occidentales, hasta este momento no han logrado descifrar la etiología de pauka/krisi siknis, han realizado los intentos, sin embargo, los que han logrado la sanción a este problema de salud son los sanadores tradicionales utilizando plantas medicinales y rituales ancestrales que solo ellos conocen. 
Es menester que, desde las autoridades del ministerio de salud pública convencional, se reconozca y comprenda que existen otras formas de enfermarse y que están relacionadas con la idiosincrasia del pueblo indígena miskitu, por ende, considero que solo a través de estrategias de coordinación y armonía entre los sanadores tradicionales y el ministerio de salud, se contribuirá en evitar los brotes de un gran número de personas afectadas por pauka/krisi siknis.

Desde el siglo XVI, la medicina fue consolidándose paulatinamente como una medicina de tradición científica y, como toda ciencia, siempre se erigió como la única y verdadera detentora del conocimiento. Desde entonces ha impuesto la primacía de sus propias nociones sobre la salud y la enfermedad, negando cualquier otro saber médico y desautorizando a otros sistemas terapéuticos de tradición milenaria, patrimonio cultural intangible de los pueblos originarios. (Martínez,S/F, p., 8).

Así también desde la época de la llegada de los misioneros cristianos, las iglesias han luchado contra estas creencias y prácticas de los diversos rituales de sanación. Ante estos acontecimientos los indígenas se vieron obligados a mantener en secreto sus conocimientos. Helms (1971) describe "curan en secreto utilizando remedios naturales como plantas medicinales y hechizan a otros por venganza o para ganar el amor de alguien, (p.182-186).

En la praxis terapéutica, la acción ritual y el concomitante manejo del poder es continuo. La cura involucra a deidades y a seres humanos poderosos, la calificación del espacio y del tiempo. El terapeuta se enfrenta a brujos causantes de daños, a ambientes que provocan enfermedades y a deidades, algunas decididamente negativas y otras que, aunque más benignas, provocan dolencias y otros perjuicios como represalia a las ofensas y faltas que se cometen. (Idoyaga y Sarudiansky, 2011, p. 327).

Por ende, se concluye manifestando la importancia de continuar avanzando en la búsqueda de otros fenómenos enfocados como misteriosos y que también causan problemas de salud en los humanos y sus formas de sanación, mismos que muchas veces permanece en secreto en los pueblos indígenas.

\section{Lista de referencia}

Conzemius-Edward. (1932). Estudio Etnográfico sobre los indios Miskitos y Sumos de Honduras y Nicaragua. Título origina Etnographical Survey of the Miskito and Sumu of Honduras and Nicaragua. Smith sonian Institution Bureau fo American Ethnology Bulletin 106. United States Printing Office Washington: 1932. Traducción al español y presentación de Jaime Incer 1984. ISBN 9977901-04. San José Costa Rica. 
Dennis-Philip A. (1999) Grisi Siknis entre los miskitos. Revista Wani No.24. Recuperado en:http://revistas.bicu.edu.ni/index.php/wani/article/view/303

Dennis Philip, A. (S/F). Grisi siknis entre los miskitus. Revista Wani de la Universidad Centro Americana (UCA) de Nicaragua.

Davis-Sandra, et al. Universidad URACCAN, (2006). Algo Anda Mal. El Bla o Wakni en el Río Coco.

Espinoza Melesio, P. (2001). Parentesco, residencia y grupo doméstico de los miskitos: los casos de Santa Martha y Auhya Pihni. Recuperado en: http://biblioteca.clacso. edu.ar/clacso/becas/20101111095222/espinoza.pdf

Fagoth A.R., et al. (1998). Wan Kaina Kulkaia, Armonizando con Nuestro Entorno.

Fagetti, A., et.al. (2011). Iniciaciones, Trances, Sueños... Investigaciones sobre el Chamanismo en México. Recuperado en: http://www.scielo.org.mx/pdf/rpfd/ v6n12/1870-4115-rpfd-6-12-275.pdf

Giovanna Cárdenas Cynthia, Pesantes Amalia María y Rodríguez Alfredo (2017). Interculturalidad en salud: reflexiones a partir de una experiencia indigena en la Amazonía peruana. Recuperado en: https://doi.org/10.1880o/ anthropologica.201702.007.

Gotz von Houwald (2003). Mayangna. Apuntes sobre la historia de los indígenas sumu en CA. ISBN 99924-53-15-X.

García, Claudia (2002). Hibridación, interacción social y adaptación cultural en la Costa de Mosquitos, siglos XVII y XVIII. Recuperado en http://estudiosamericanos. revistas.csic.es/index.php/estudiosamericanos/article/view/181/185.

Helms Mary, (1971). Asang: Adaptación al contacto cultural en una sociedad miskita, Instituto Indigenista Interamericano.

Henríquez-Levas, et al. (2016). Percepción y prácticas de atención del Grisi siknis en la comunidad de krin krin, Waspam, Río Coco. Recuperado en: https://revistas. uraccan.edu.ni/index.php/Caribe/article/view/383

Hales et al., (1985), tratado de psiquiatría.

Idoyaga Molina Anatilde y Mercedes Sarudiansky (Las Medicinas Tradicionales en el noroeste argentino. Reflexiones sobre tradiciones académicas, saberes populares, terapias rituales y fragmentos de creencias indígenas. 
Jamieson Mark. Wani No. 57 (2013). El lenguaje y el arte del desconsuelo entre los miskitus. Departamento de Antropología Social, Universidad de Manchester. Recuperado en: revistasnicaragua.net.ni/index.php/wani/article/ view/1627/1574.

Langdon EJ, Wiik FB. (2010). Antropología, salud y enfermedad: una introducción al concepto de cultura aplicado a las ciencias de la salud. Recuperado en: http:// www.scielo.br/pdf/rlae/v18n3/es_23.pdf.

Martínez Cruz, Jessica (S/F). jEste conocimiento cuenta! Armonía y espiritualidad en el pensamiento crítico miskito.

Organización Mundial de la salud (2019). Salud Universal en el Siglo XXI: 40 años de Alma-Ata. Informe de la Comisión de Alto Nivel.

Organización Mundial de la Salud (2014). Estrategia de la OMS sobre Medicina Tradicional 2014-2023.

Pérez-Chiriboga I. (2002). Espíritus de vida y muerte. Los Miskitus Hondureños en épocas de guerra.

Pichot-Pierre, et al. (1995). DSM-IV Manual diagnóstico y estadístico de los trastornos mentales.

Romero-Arrechavala, J., et al. (2007). Historia de la Costa Caribe de Nicaragua. Un enfoque regional. Impreso en: grupo editorial acento S.A. Recuperado en: http:// catalogo.ihnca.edu.ni/cgi-bin/koha/opac- detail.pl?biblionumber=59511. ISBN: 978-99924-66-21-6.

Venegas-María, D., (2016). Alienated Affliction: The Politics of Grisi Siknis Experience In Nicaragua. University of Pittsburgh. The Dietrich School Of Arts And Sciences. http://d-scholarship.pitt.edu/30633/URI: http://d-scholarship.pitt.edu/id/ eprint/30633.

Von-Houwald, G.F., (2003). Mayangana, Apuntes sobre la historia de los indígenas Sumu en Centroamérica. Contribuciones a la etnología. Colección cultural de Centro América. Serie etnología No. 1. ISBN 99924-53-15-X.

Wedel J. (2012). Involuntary mass spirit possession among the Miskitu. Recuperado en: https://www.researchgate.net/publication/228489007_Bridging_the_Gap_between_Western_and_Indigenous_Medicine_in_Eastern_Nicaragua. 